\title{
Serum amylin level in Behçet's disease and relation to parameters of metabolic syndrome and disease activity
}

\author{
Shimaa M. Abdelwhab ${ }^{a}$, Omima Z. Shehata ${ }^{a}$, Yousry A. El Magd ${ }^{b}$, \\ Amal Kamal ${ }^{\mathrm{b}}$
}

\author{
Departments of ${ }^{a}$ Rheumatology and \\ Rehabilitation, ${ }^{b}$ Biochemistry, Zagazig \\ University, Zagazig, Egypt \\ Correspondence to Shimaa Mostafa \\ Abdelwhab, Lecturer, Department of \\ Rheumatology and Rehabilitation, Zagazig \\ University, Zagazig, Egypt. \\ e-mail: abuyehia@gmail.com \\ Received 23 August 2017 \\ Accepted 16 October 2017
}

Egyptian Rheumatology \& Rehabilitation 2018, 45:112-116

\begin{abstract}
Background
Insulin resistance is found in Behçet's disease and associated with the development of metabolic syndrome. Our study explored whether amylin, which is involved in insulin resistance and development of metabolic syndrome, is observed in patients with Behçet's disease.

Aim

Our aim was to assess serum levels of amylin in patients with Behçet's disease and evaluate its relation to metabolic syndrome.

Patients and methods

Thirty Behçet's disease patients and 30 controls matched by age, sex, and BMI were studied. The disease activity was assessed using the Behçet's disease Current Activity Form score. Serum amylin level was studied using the enzymelinked immunosorbent assay method.

Results

Our result showed that $11(36.6 \%)$ patients under study were diagnosed with the metabolic syndrome, and there were significant differences between Behçet's cases and control groups in cholesterol, triglyceride, and low-density lipoprotein levels $(P<0.05)$. Amylin level was $392.2 \mu \mathrm{g} / \mathrm{dl}$ in Behçet's cases compared with $659.9 \mu \mathrm{g} /$ $\mathrm{dl}$ in the control group. The difference was statistically significant at $P$ value less than 0.05 . There was a negative correlation between amylin levels and parameters of metabolic syndrome, a significant difference in amylin levels between Behçet's patients with an active manifestation and patient with an inactive manifestation.

Conclusion

Amylin serum levels were significantly lower in patients with Behçet's disease, indicating that this hormone could be a factor for the development of metabolic syndrome in Behçet's disease.
\end{abstract}

\author{
Keywords: \\ amylin, Behcet, metabolic syndrome \\ Egypt Rheumatol Rehabil 45:112-116 \\ () 2018 Egyptian Society for Rheumatology and Rehabilitation \\ 1110-161X
}

\section{Introduction}

Metabolic syndrome (MetS) is a major health problem. MetS affects about one in four individuals in the Middle East, characterized by hyperglycemia, hyperlipidemia, hypertension, and obesity, and leads to cardiovascular risk and nonalcoholic fatty liver disease [1].

Behçet's disease (BD) is a multisystemic disease. BD may induce the development of MetS [2]. BD results in a higher ratio of MetS. MetS tends to increase with the activity of the disease. Cardiovascular event-related risk factors must be taken into account in BD [3].

Amylin or Islet amyloid polypeptide is a 37-residue peptide hormone. It is cosecreted with insulin from the pancreatic $\beta$-cells. Amylin plays a role in glycemic regulation; weight controls to a lower mass by promoting satiety [4].
Amylin is associated with inflammatory markers and MetS. The amylin-MetS association is independent of established risk factors of MetS, including obesity, inflammatory markers, and insulin resistance (IR) [5].

\section{Patients and methods Patients}

This study was carried out in the Rheumatology and Rehabilitation Department, Faculty of Medicine, and written consents were obtained from the patients.

The study was carried out according to the Zagazig University Ethical Committee and was approved by the Ethics Committee of Zagazig University.

This is an open access journal, and articles are distributed under the terms of the Creative Commons Attribution-NonCommercial-ShareAlike 4.0 License, which allows others to remix, tweak, and build upon the work non-commercially, as long as appropriate credit is given and the new creations are licensed under the identical terms. 
Sixty participants were divided into two groups. Group I: BD group, which included 30 individuals with $\mathrm{BD}$, diagnosed according to the new International Criteria for Behçet's disease [6]. There were 22 men and eight women, ranging in age from 28 to 64 years. Group ?I: the control group included individuals who were apparently healthy and were age, sex, and BMI matched with the patients in the $\mathrm{BD}$ group. Their ages ranged from 21 to 52 years.

\section{Data collection}

The age, sex, and patients and controls were determined. Height, weight, BMI, waist circumference (which was measured midway between the lower costal margin and the iliac crest), blood pressure after 5 min of rest, fasting blood glucose, triglycerides (TG), total cholesterol, lowdensity lipoprotein (LDL), very LDL, high-density lipoprotein, erythrocyte sedimentation rate, and C-reactive protein levels were measured in the study patients and these patients were further investigated for age at onset of the disease, duration of the disease, clinical manifestations of the disease, and medications used for BD. Patients were assessed using Behçet's Disease Current Activity Form 2006 [7]. All scoring depends on the symptoms present over the 4 weeks before assessment. Patients who fulfilled three of the above five criteria of the Adult Treatment Panel-III Criteria [8] were considered to have the MetS.

\section{Measurement of amylin in the serum Principle of the method}

Assay the amylin in the sample of human's serum. The kit (cat.no:CK-E10377) uses a double-antibody sandwich enzyme-linked immunosorbent assay to assay the level of human amylin in samples. Amylin is added to a monoclonal antibody Enzyme well that is precoated with the human amylin monoclonal antibody.

\section{Statistical design}

The collected data were entered and analyzed by a computer using the statistical package of social sciences, version 20 (SPSS Inc., Chicago, Illinois, USA). Qualitative data ( $n$ ) number of each observation at. (\%) percentage of the observation to all category or order. Quantitative data mean, range, SD was used.

$\chi^{2}$ and Fisher's exact tests were used for comparisons of categorical data. Differences in the means of continuous measurements were tested using Student's t-test. $P$ values less than 0.05 were considered to be statistically significant. The Mann-Whitney test used when required. The correlation coefficient $(r)$ measures the correlation between quantitative continues variables.

\section{Results}

Table 1 shows that there were no significant differences between Behçet's cases and control groups in the mean age, BMI, or sex $(P>0.05)$. There were significant differences between Behçet's cases and control groups in cholesterol, TG, and LDL levels $(P<0.05)$.

Table 2 shows that a total of 11 (36.6\%) patients under study were diagnosed with the MetS and three (10\%) controls were diagnosed with the MetS.

Table 3 shows that the mean amylin level was $392.2 \mu \mathrm{g} / \mathrm{dl}$ in Behçet's cases compared with $659.9 \mu \mathrm{g} / \mathrm{dl}$ in the control group. The difference was statistically significant $(P<0.05)$.

Table 1 Demographic and laboratory and the data of mean metabolic parameters of the participants

\begin{tabular}{|c|c|c|c|c|c|}
\hline & Behçet's (mean $\pm S D)$ & Control (mean $\pm S D)$ & $t$-Value & $P$-value & Significance \\
\hline & $35 \pm 9.4$ & $34.8 \pm 8.9$ & 0.07 & 0.9 & NS \\
\hline BMI $\left(\mathrm{kg} / \mathrm{m}^{2}\right)$ & $23.7 \pm 1.3$ & $24.3 \pm 2$ & 1.5 & 0.13 & NS \\
\hline \multicolumn{6}{|l|}{ Sex } \\
\hline Male & 22 & 15 & $3.4^{*}$ & 0.06 & NS \\
\hline Female & 8 & 15 & & & \\
\hline \multicolumn{6}{|l|}{ Duration of Behçet's/year } \\
\hline Mean \pm SD & $6.4 \pm 3.4$ & & & & \\
\hline Range & $1-15$ & & & & \\
\hline Systolic blood pressure & $126 \pm 19$ & $129 \pm 21$ & 0.5 & 0.6 & NS \\
\hline Diastolic blood pressure & $81 \pm 11$ & $79 \pm 10$ & 0.6 & 0.5 & NS \\
\hline Fasting blood sugar & $156 \pm 69$ & $118 \pm 42$ & 2.5 & 0.01 & $\mathrm{~S}$ \\
\hline Cholesterol & $234 \pm 35$ & $156 \pm 34$ & 8.6 & 0.00 & $S$ \\
\hline Triglyceride & $157 \pm 81$ & $116 \pm 55$ & 2.2 & 0.02 & $S$ \\
\hline HDL & $46 \pm 21$ & $58 \pm 14$ & 1.5 & 0.13 & NS \\
\hline LDL & $155 \pm 41$ & $94 \pm 32$ & 6.4 & 0.00 & $\mathrm{~S}$ \\
\hline
\end{tabular}

HDL, high-density lipoprotein; LDL, low-density lipoprotein. ${ }^{*} P$ value significant less .05. 
Table 4 shows significant differences in amylin levels between Behçet's patients with an active manifestation and patients with an inactive manifestation.

Table 5 shows that there is a significant negative correlation between serum amylin levels among Behçet's cases and blood cholesterol levels, LDL levels, TG levels, and duration of BD.

Table 6 shows a significant negative correlation between corticosteroid dose and amylin levels and a

Table 2 The Adult Treatment Panel III criteria of patients and controls

\begin{tabular}{ccc}
\hline & Present & Positive metabolic syndrome \\
\hline Waist circumference & $(>88 \mathrm{~cm}$ in women and $>102 \mathrm{~cm}$ in men $)$ \\
Patients & 16 & 11 \\
Controls & 10 & 3 \\
TG $>150 \mathrm{mg} / \mathrm{dl}$ & & \\
Patients & 12 & 11 \\
Controls & 5 & 2 \\
HDL level $<40$ and $50 \mathrm{mg} / \mathrm{dl}$ in men and women & \\
Patients & 8 & 8 \\
Controls & 6 & 4 \\
Blood pressure $(\mathrm{SBP}>130 \mathrm{mmHg}$ and DBP $>80 \mathrm{mmHg})$ \\
Patients & 14 & 12 \\
Controls & 8 & 3 \\
Blood sugar $>100 \mathrm{mg} / \mathrm{dl}$ & \\
Patients & 14 & 10 \\
Controls & 8 & 2 \\
\hline
\end{tabular}

DBP, diastolic blood pressure; HDL, high-density lipoprotein; LDL, low-density lipoprotein; SBP, systolic blood pressure; TG, triglyceride. positive significant correlation between cortisone dose and serum cholesterol levels, LDL levels, TG levels, and activity score among Behçet's cases (Figs 1 and 2).

\section{Discussion}

$\mathrm{BD}$ is a multisystemic inflammatory disease and the expression of proinflammatory cytokines (tumor necrosis factor- $\alpha$, interleukin- 6 ) is associated with IR; the cumulative effects of inflammation lead to the development of MetS [9].

The pancreatic peptide amylin is cosecreted with insulin and is best known for its role as a satiation hormone. Amylin levels increase postprandially,

\section{Figure 1}

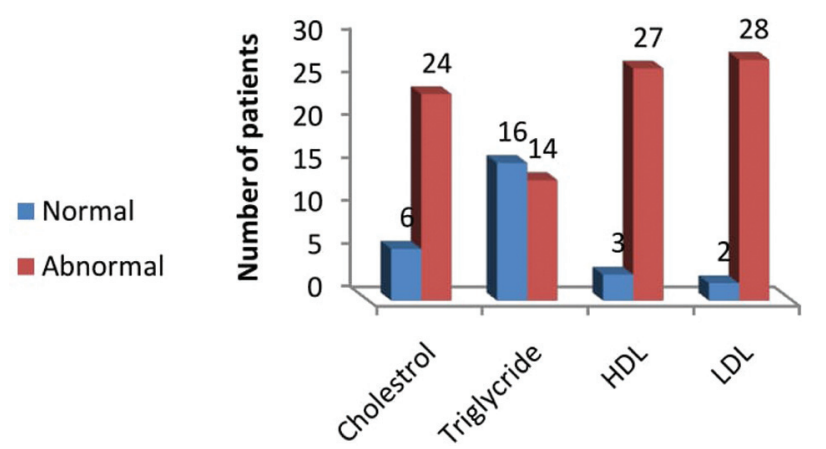

Number of Behçet's disease patients with normal and abnormal levels of blood cholesterol, triglyceride, low-density lipoprotein (LDL), and high-density lipoprotein (HDL).

Table 3 Comparison of the serum levels of amylin of Behçet's disease cases and the control group

\begin{tabular}{|c|c|c|c|c|c|}
\hline & Behçet's patients $(N=30)$ & Control group $(N=30)$ & Mann-Whitney & $P$-value & Significance \\
\hline \multicolumn{6}{|l|}{ Amylin } \\
\hline Mean \pm SD & $392.2 \pm 3.5$ & $659.9 \pm 482$ & - & 0.034 & Significant \\
\hline Median & 296 & 644.7 & & & \\
\hline Range & 4.38-903.12 & $15.19-1524.93$ & & & \\
\hline
\end{tabular}

Table 4 Amylin among active and inactive Behçet's disease patients

\begin{tabular}{lcc}
\hline Behçet's patients & $N[$ mean \pm SD (range) & $P^{*}$ \\
\hline Active group $($ score $\geq 6)$ & $25[306 \pm 256(4.38-755)]$ & 0.001 \\
Inactive group $<6$ & $5[875 \pm 76.6(801-903.12)]$ & \\
\hline
\end{tabular}

${ }^{*} P$ value significant less .05 .

Table 5 Correlation between amylin levels $(\mu \mathrm{g} / \mathrm{dl})$ and parameters of metabolic syndrome and duration of disease among Behçet's disease cases

\begin{tabular}{lcc}
\hline Amylin & Pearson's correlation coefficient $(r)$ & $P$ \\
\hline Cholesterol & -0.39 & 0.02 \\
LDL & -0.32 & 0.01 \\
HDL & 0.014 & 0.91 \\
Triglyceride & -0.4 & 0.02 \\
Fasting blood sugar & -0.73 & 0.0 \\
Duration of disease & -0.58 & 0.001 \\
\hline
\end{tabular}

HDL, high-density lipoprotein; LDL, low-density lipoprotein. 
Table 6 Correlation between cortisone, amylin, activity score, and metabolic syndrome parameters

\begin{tabular}{lccccccc}
\hline Cortisone & Amylin & Serum cholesterol & LDL & HDL & Triglyceride & RBS & Activity score \\
\hline$r$ & -0.7 & 0.5 & 0.36 & -0.33 & 0.38 & 0.5 & 0.6 \\
$P$ & 0.00 & 0.004 & 0.04 & 0.07 & 0.036 & 0.004 & 0.000 \\
\hline
\end{tabular}

HDL, high-density lipoprotein; LDL, low-density lipoprotein; RBS, random blood sugar.

Figure 2

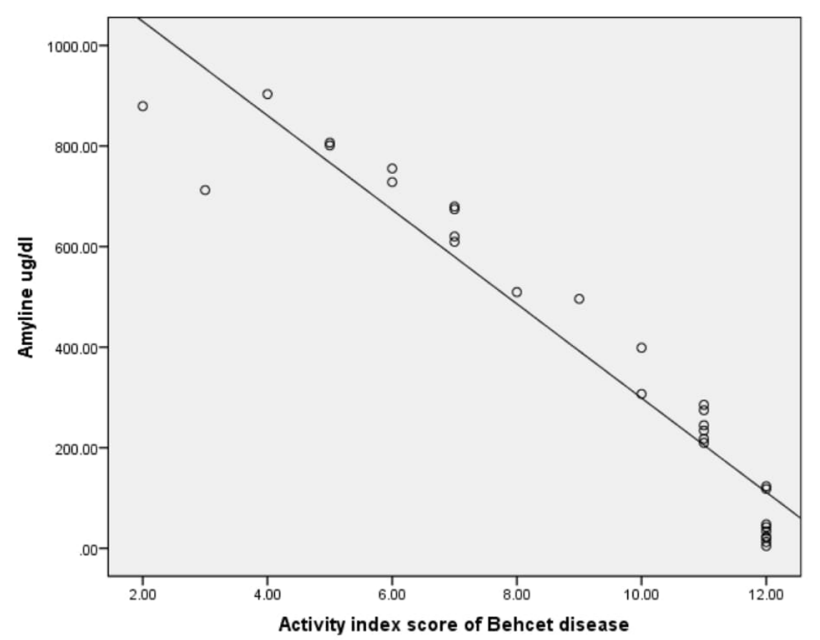

There is a significantly negative correlation between the activity score of Behçet's disease and amylin levels $(r=-0.96, P=0.000)$.

whereas amylin analog injection supresses food intake in rodents; in humans with type 2 diabetes, an injection of the amylin analog improves insulin senstivity and induces weight loss [9].

There are few studies on the role of amylin in BD and its association with MetS in BD. This study evaluated the importance of amylin in BD and confirmed the significant correlation of amylin with disease activity and MetS.

The results of our study suggest that MetS is more in BD than ordinary people.

In another Egyptian study, it was found that MetS was present in $31.8 \%$ of patients versus $6.67 \%$ of controls [10].

A research was published in 2013 in Turkey by Yalç et al. [11]. The study examined the prevalence of the MetS in BD patients. The prevalence of the MetS in the $\mathrm{BD}$ group and the control group was 35.4 and $20 \%$, respectively [11]. In Iranian studies, the prevalence of the METS in patients with BD was not higher than that in healthy individuals $[12,13]$.

We found that amylin levels were lower in patients with $\mathrm{BD}$; similar results were found in the study of Dogan et al. [14].
Our results showed that there was a significant negative correlation between serum amylin and disease activity. This was not in agreement with the results of the study of Dogan et al. [14].

Our results showed that there is a significant negative correlation between serum amylin levels among Behçet's patients and blood cholesterol, LDL, and TG levels. This is not in agreement with the results of a Chinese study. It was found in that study that there was a positive correlation between amylin and MetS in the Chinese patients, and that this result was found irrespective of risk factors such as obesity, inflammatory markers, and IR [5]. This may be explained by abnormal amylin production, which is a feature peripheral pathology both in the early (prediabetic) and in the late phases of type 2 diabetes mellitus. Hyperamylinemia is found in the early phase and hypoamylinemia is found in late-phase conditions, and correspond with hyperinsulinemia and hypoinsulinemia, respectively [13]; our results also support trials of synthetic amylin to modulate TG levels.

Our results found a significantly negative correlation between corticosteroid dose and amylin levels. This could be explained by the fact that corticosteroid treatment directly affects B-cell function. Although less explored than insulin release, these steroids also affect the secretion of other islet hormones with important roles in glucose homeostasis, such as amylin. Alterations in islet hormonal secretion can exacerbate the diabetogenic actions of glucocorticoids [15].

\section{Conclusion}

Amylin levels were lower in BD patients compared with the healthy controls. There was a negative correlation between amylin levels and parameters of Mets. Synthetic serum amylin may be a solution for the treatment of MetS in BD.

\section{Financial support and sponsorship}

Nil.

\section{Conflicts of interest}

There are no conflicts of interest. 


\section{References}

1 Grundy SM, Cleeman JI, Daniels SR, Donato KA, Eckel RH, et al. Diagnosis and management of the metabolic syndrome: an American Heart Association/National Heart, Lung, and Blood Institute Scientific Statement. Circulation 2005; 112:2735-2752.

2 Alireza K, Mehrzad H, Noei M. Metabolic syndrome in Behcet's disease. International Conference on Behcet's Disease; 2014; Emamreza, Tabriz, Iran. pp. 18-20.

3 Ulaşoğlu C, Mesci B, Karagöz E, Karagöz V, Akın Y, Öztürk H, et al. Frequency of metabolic syndrome and nonalcoholic fatty liver in Behçet's disease. Nobel Med J 2013; 9:35-42.

4 Higham CE, Hull RL, Lawrie L, Shennan KI, Morris JF, Birch NP, et al. Processing of synthetic pro-islet amyloid polypeptide (prolAPP) amylin by recombinant prohormone convertase enzymes, PC2 and PC3, in vitro. Eur J Biochem 2005; 267:4998-5004.

5 Hou X, Sun L, Li Z, Mou H, Yu Z, Li H, et al. Associations of amylin with inflammatory markers and metabolic syndrome in apparently healthy Chinese. PLoS One 2011; 6:e24815.

6 Davatchi F, Assaad KS, Calamia KT. International Team for the Revision of the International Criteria for Behcet's Disease: a collaborative study of 27 countries on the sensitivity and specificity of the new criteria. J Eur Acad Dermatol Venereol 2014; 28:338-347.

7 Forbess C, Swearingen C, Yazici Y. Behçet's Syndrome Activity Score (BSAS): a new disease activity assessment tool, composed of patientderived measures only, is strongly correlated with the Behçet's Disease Current Activity Form (BDCAF). Arthritis Rheum 2008; 58(Suppl):S854.

8 Beilby J. Definition of metabolic syndrome: report of the National Heart, Lung, and Blood Institute/American Heart Association Conference on Scientific Issues Related to Definition. Clin Biochem Rev 2004; 25 : 195-198.

9 Lutz TA, Meyer U. Amylin at the interface between metabolic and neurodegenerative disorders. Front Neurosci 2015; 9:216.

10 Abou-Raya A, Abou-Raya S, Helmii M. Metabolic syndrome in Behçet's disease: association with disease activity and systemic inflammation. Ann Rheum Dis 2014; 73:993.

11 Yalç NB, Gür G, Artüz F, All N. Prevalence of metabolic syndrome in Behçet disease : a case-control study in Turkey. Am J Clin Dermatol 2013; 14: $421-425$.

12 Sadrbafoghi SM, Salari M, Rafiee M, Namayandeh SM, Abdoli AM, Karimi $\mathrm{M}$, et al. Prevalence and criteria of metabolic syndrome in an urban population: Yazd Healthy Heart Project. Tehran Univ Med J 2006; 64: 90-96.

13 Azizi F, Etemadi A, Salehi P, Zahedi Asl S. Prevalence of metabolic syndrome in an urban population: Tehran Lipid and Glucose Study. Tehran Univ Med J 2003; 61:389-399.

14 Dogan FB, Cicek D, Aydin S, Dertlioglu SB, Halisdemir N, Ucak H, et al. Serum preptin and amylin values in psoriasis vulgaris and Behcet's patients. J Clin Lab Anal 2016; 30:165-168.

15 Rafacho A, Ortsäter H, Nadal A, Quesada I. Glucocorticoid treatment and endocrine pancreas function: implications for glucose homeostasis, insulin resistance and diabetes. J Endocrinol 2014; 223: R49-R62. 